\title{
Intoxicación plúmbica crónica y alteraciones del crecimiento y desarrollo cognitivo-emocional en niños
}

\author{
JULIÁN VEGA ${ }^{1}$, JUAN DE COLL ${ }^{1}$, DORIS KATEKARU ${ }^{1}$, JORGE LERMO ${ }^{1}$, JUAN ESCOBAR ${ }^{1}$, MIRIAM DÍAZ ${ }^{1}$, \\ VÍCTOR BERROCAL ${ }^{1}$, JENNY GÓMEZ ${ }^{1}$, GIANCARLO DÍAZ ${ }^{1}$, ÁLVARO ARROYO ${ }^{1}$, JOSÉ CASTRO ${ }^{2}$ \\ ${ }^{1}$ Alumnos de la Escuela Académico Profesional de Medicina Humana. ${ }^{2}$ Director Ejecutivo de Evaluación de \\ Cooperación Internacional. ${ }^{1,2}$ Facultad de Medicina de San Fernando, UNMSM.
}

\begin{abstract}
RESUMEN
OBJETIVO: Determinar la asociación entre intoxicación plúmbica crónica y alteraciones del crecimiento y desarrollo cognitivo-emocional en niños del colegio "María Reiche"- Callao. DISEÑO: Estudio observacional de corte transversal. MATERIAL Y MÉTODOS: A 70 niños de 8 a 12 años de edad del C.E. No 5045 "María Reiche" del A.A.H.H. "Puerto Nuevo"- Callao, que participaron en el estudio de dosaje de plomo en sangre hecho por la Dirección General de Salud Ambiental en 1999, se les aplicó pruebas de seguimiento de instrucciones, habilidades motoras, comunicativas, manipulativas, académicas y la lista de chequeo de ansiedad. Se midió el coeficiente intelectual e índice de masa corporal. Para el procesamiento de los datos se empleó las pruebas chi-cuadrado, t de Student, correlación y regresión lineal simple, usando el paquete estadístico SPSS versión 11.0, con un nivel de confianza de $95 \%$. RESULTADOS: Los niveles de plomo en sangre y los grados de ansiedad se relacionaron significativamente mediante la prueba chi-cuadrado $(p=0,025)$; se encontró diferencias significativas entre las medias del puntaje de la lista de chequeo de ansiedad y la categorización de los niveles de plomo, mediante la prueba $t$ de Student $(p=0,031)$. La relación entre los niveles de plomo en sangre y el coeficiente intelectual, las habilidades académicas, motoras, comunicativas, manipulativas, seguimiento de instrucciones e índice de masa corporal no resultaron significativos. CONCLUSIONES: Se encontró asociación significativa entre la intoxicación plúmbica crónica y el grado de ansiedad (desarrollo emocional). Sin embargo, no se encontró asociación con el crecimiento ni con el desarrollo cognitivo. Palabras clave: Envenenamiento por plomo; exposición a riesgos ambientales; inteligencia; desarrollo infantil.
\end{abstract}

\section{CHRONIC LEAD INTOXICATION AND GROWTH AND COGNITIVE-EMOTIONAL DEVELOPMENT IN CHILDREN \\ SUMMARY}

OBJECTIVE: To determine the association between chronic lead intoxication and growth and cognitiveemotional development alterations in children at "María Reiche" school - Callao. DESIGN: Crossectional study. MATERIAL AND METHODS: To 70 children 8 to 12 year-old from "María Reiche" school (A.A.H.H. "Puerto Nuevo"- Callao), who had participated in a survey blood lead screening (General Direction of Environmental Health, in 1999), we applied instructions monitoring tests, communicative, motor, manipulator, and academic abilities test as well as anxiety checkup list. The intellectual coefficient and BMI (body mass index) were measured. Chi square, $t$-Student and simple lineal correlation tests were employed for prosecution of data using the statistical package SPSS version 11.0 with a $95 \%$ confidence level. RESULTS: By chi square test there was a significant $(p=0,025)$ relation between blood lead levels and degrees of anxiety. There was significant difference between anxiety check-up list score averages and categorization of lead levels by t-Student test $(p=0,031)$. The relation between blood lead levels and intellectual coefficient, as well as communicative, motor, academic, manipulator abilities, monitoring of instructions and body mass index did not turn out to be significant. CONCLUSION: There is an association between chronic lead intoxication and anxiety degree (emotional development). However, there was no association with growth or cognitive development.

Key words: Lead poisoning; environmental exposure; intelligence; child development.

Correspondencia:

Dr. José Castro Quiroz

Los Pinos 150. Lima 31, Perú

E-mail: jcastroq@minsa.gob.pe 


\section{INTRODUCCIÓN}

El plomo es un metal pesado cuyo peso atómico es 207,2, que no cumple algún papel en la fisiología humana $\left({ }^{1-4}\right)$. Su mecanismo de toxicidad consiste en reemplazar al calcio durante el transporte iónico $\left({ }^{5}\right)$. Los adultos absorben entre $5 \%$ y $10 \%$ del plomo ingerido; sin embargo, sólo retienen el $5 \%$, mientras que los niños, debido a su rápido metabolismo $\left({ }^{6}\right)$, absorben el $40 \%$ y retienen $30 \%\left({ }^{7}\right)$. La acumulación de plomo en el organismo se da principalmente en el hueso $\left({ }^{6}\right)$, por lo que es considerado una fuente de exposición endógena $\left({ }^{8}\right)$; en adultos, esta acumulación es cercana a $95 \%$, mientras que en niños la cifra es próxima a $70 \%\left({ }^{8,9}\right)$. La vida media del plomo en los tejidos blandos -como el riñón, cerebro e hígado- oscila entre 20 y 30 días $\left({ }^{1}\right)$; en los glóbulos rojos es aproximadamente 35 días y en el hueso varía de 5 a 30 años $\left({ }^{1,8,10}\right)$. En la intoxicación plúmbica crónica $\left({ }^{11}\right)$, la población infantil es la de mayor riesgo, ya que sus tejidos blandos se encuentran en pleno desarrollo $\left({ }^{2,12-14}\right)$. En los niños, el sistema nervioso es el principal tejido afectado por el plomo, incluso a concentraciones bajas, depositándose principalmente en la sustancia gris y los núcleos basales $\left({ }^{3,4}\right)$. La neurotoxicidad que produce conlleva a severos trastornos de las funciones cognitivas, que se expresan en problemas de aprendizaje y conducta, como irritabili-

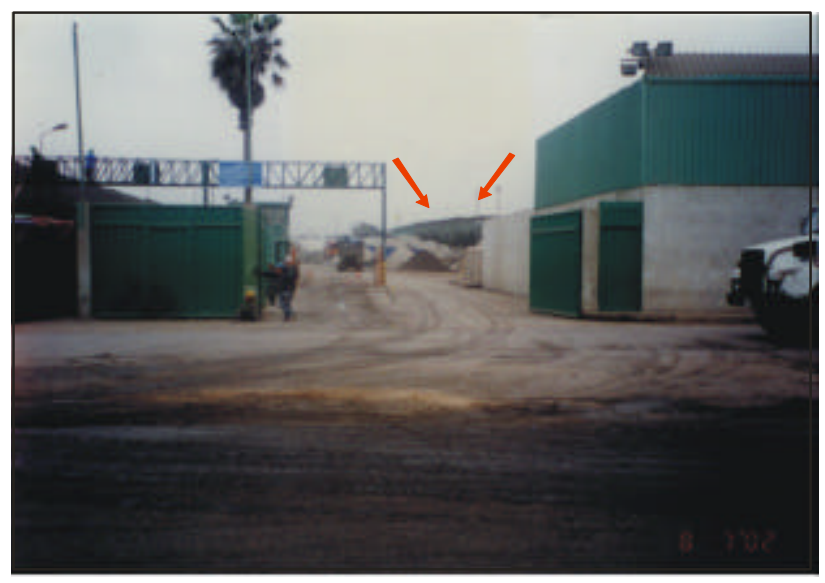

Figura 1.- Vista panorámica donde se observa montículos de plomo que colindan con el C.E. 5045 "María Reiche". dad, hiperactividad, cefalea, disminución de la agudeza visual, retraso mental, alteraciones del lenguaje y bajo rendimiento escolar $\left({ }^{2,15-17}\right)$.

Los niveles de plomo en sangre admitidos como aceptables han sido reformulados muchas veces, a medida que se ha ampliado el conocimiento de los trastornos que produce este metal en el hombre. En la actualidad, el Centro de Control de Enfermedades de los Estados Unidos aconseja que los habitantes, en general, deben tener niveles de plomo en sangre que se encuentren por debajo de los $10 \mu \mathrm{g} / \mathrm{dL}\left({ }^{18}\right)$. En los niños, los síntomas suelen aparecer a partir de $\operatorname{los} 30 \mu \mathrm{g} / \mathrm{dL}\left({ }^{19}\right)$.

La Dirección General de Salud Ambiental (DIGESA) encontró en el Callao que los niños que acuden a los colegios "Guadalupe" y "María Reiche" (ubicados cerca de una extensa área de almacenamiento de minerales) $\left({ }^{20}\right)$ registraron una media de plomo en sangre de 40,7 $\mu \mathrm{g} / \mathrm{dL}$, mientras que en los niños de otras escuelas el nivel fue $7,5 \mu \mathrm{g} / \mathrm{dL}\left({ }^{19,20}\right)$. Observaron que el vivir o estudiar cerca a los depósitos de plomo aumenta en casi 18 veces el riesgo de tener valores elevados de plomo en sangre $\left({ }^{19}\right)$ (Figura 1 ).

Existe una alta prevalencia de intoxicación por plomo $(93,4 \%)$ en los niños del A.A.H.H. "Puerto Nuevo", donde se ubica el colegio "María

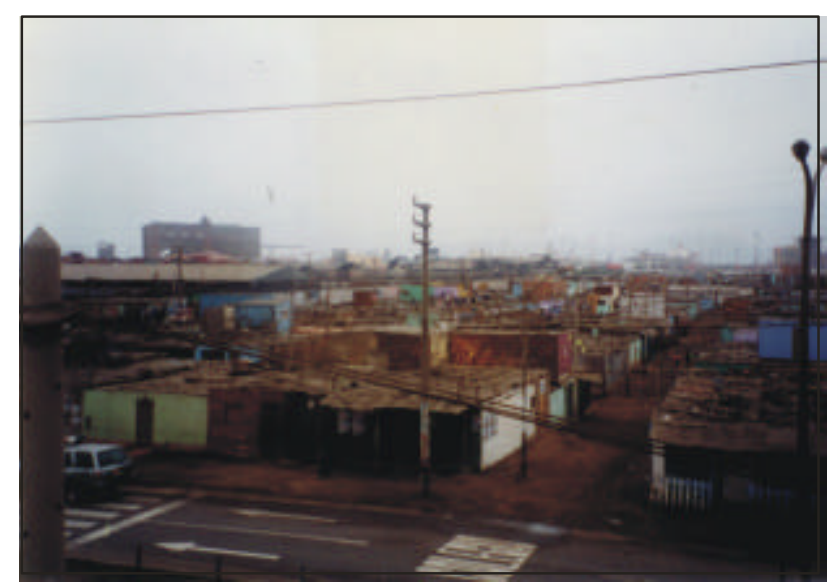

Figura 2.- Vista panorámica del A.A.H.H. "Puerto Nuevo" tomada desde el C.E. 5045 "María Reiche”. 
Reiche", siendo en los primeros 24 meses de vida cuando se observa la mayor velocidad de acumulación de plomo en el organismo $\left({ }^{21}\right)$ (Figura 2).

Dada esta situación, nos planteamos desarrollar la presente investigación, debido a las graves consecuencias que la exposición crónica al plomo produce en los niños.

El objetivo fue determinar la asociación entre intoxicación plúmbica crónica y alteraciones del crecimiento y desarrollo cognitivo-emocional en niños del colegio "María Reiche"- Callao, midiendo el coeficiente intelectual, evaluando el seguimiento de instrucciones, las habilidades motoras, comunicativas, manipulativas y académicas, determinando el índice de masa corporal y midiendo el grado de ansiedad.

\section{MATERIAL Y MÉTODOS}

El área de estudio fue el C.E. No 5045 María Reiche, centro de educación primaria ubicado en la Av. Contralmirante Mora $\mathrm{N}^{\circ} 420$, A.A.H.H "Puerto Nuevo", provincia Constitucional del Callao, que cuenta con 11 aulas distribuidas en dos plantas.

El tipo de estudio es observacional y de corte transversal. La población de estudio estuvo conformada por la totalidad del alumnado (271 niños). El tipo de muestreo fue no probabilístico. Se tomó como criterios de inclusión pertenecer a la lista de dosaje de plomo en sangre realizado por DIGESA en 1999, tener entre 8 y 12 años de edad, estar matriculado en el año académico 2002 de dicho colegio y residir en el A.A.H.H "Puerto Nuevo". El criterio de exclusión fue no haber aceptado participar en el estudio.

Nuestra muestra la conformaron 70 individuos. Los niños participantes se encontraban entre el $2^{\circ}$ y $6^{\circ}$ grado de educación primaria, siendo la media de sus edades 9,8 años y la desviación estándar 1,13; además, sus niveles de plomo en sangre* se encontraban entre el rango de 17 y $107 \mu \mathrm{g} / \mathrm{dL}$, con una media de $40,7 \mu \mathrm{g} / \mathrm{dL}$ y una desviación estándar de 15,2 $\mu \mathrm{g} / \mathrm{dL}$. Como método de análisis de plomo se utilizó el Lead Care (método rápido) aplicándosele a toda medición mayor a $30 \mu \mathrm{g} / \mathrm{dL}$ una prueba de absorción atómica para determinar el nivel correspondiente.

La medición del coeficiente intelectual (CI) se realizó mediante la prueba Cattell G-2 esco$\operatorname{lar}^{\dagger}$ para niños de 8 a 14 años.

Se aplicó las pruebas específicas de seguimiento de instrucciones, habilidades motoras, comunicativas, manipulativas y académicas, aprobados por la Asociación Peruana de Psicología $(\text { ASPSSI })^{\dagger}$ que miden la presencia o la ausencia, mayor o menor, de una habilidad en particular, en niños de 0 a 12 años de edad; se clasifica cada prueba en las categorías desarrollo normal, alteración leve, moderada y marcada.

Se aplicó también la lista de chequeo de ansiedad para niños de 0-12 años de edad, aprobado por el Centro Peruano de Investigaciones Psicológicas y Modificación del Comportamiento (CIPMOC) ${ }^{\dagger}$, que evalúa el ámbito emotivo; consistió en 26 ítems de alternativa múltiple, realizado en una entrevista estructurada, clasificándose en categorías de ansiedad normal, ansiedad leve y ansiedad marcada.

Luego de pesar y tallar a los niños, se calculó el índice de masa corporal (IMC), estableciéndose como normal el rango comprendido entre $15,5 \mathrm{~kg} / \mathrm{m}^{2}$ y $18,9 \mathrm{~kg} / \mathrm{m}^{2}$, datos proporcionados por el Departamento de Nutrición del Instituto Nacional de Salud del Niño.

Se evaluó la asociación entre los niveles de plomo, en dos categorías: valores menores o iguales de $30 \mathrm{ug} / \mathrm{dL}$ (nivel sub-clínico) y mayores de $30 \mu \mathrm{g} / \mathrm{dL}$ de plomo en sangre (nivel clínico).

Los resultados de las pruebas ya mencionadas fueron analizados utilizando las pruebas chi-

\footnotetext{
* Tomados de la lista de dosaje de plomo en sangre realizado por DIGESA en 1999.

$\dagger$ Obtenidas de la Facultad de Psicología de la Universidad Nacional Mayor de San Marcos.
} 
Tabla 1.- Tabla de contingencia de la categorización de los niveles de plomo en sangre y la categorización de los puntajes de la lista de chequeo de ansiedad.

\begin{tabular}{|c|c|c|c|c|c|}
\hline & & \multicolumn{3}{|c|}{$\begin{array}{l}\text { Categorización de los puntajes de } \\
\text { la lista de chequeo de ansiedad }\end{array}$} & \multirow[b]{2}{*}{ Tota } \\
\hline & & normal & alteración leve & alteración marcada & \\
\hline \multirow{3}{*}{$\begin{array}{l}\text { Categorización de los } \\
\text { niveles plomo en sangre }\end{array}$} & Grupo $1^{*}$ & 2 & 6 & 8 & 16 \\
\hline & Grupo $2^{\dagger}$ & 0 & 17 & 36 & 53 \\
\hline & Total & 2 & 23 & 44 & 69 \\
\hline
\end{tabular}

* Grupo 1: menores o iguales a $30 \mu \mathrm{g} / \mathrm{dL}$

${ }^{\dagger}$ Grupo 2: mayores a $30 \mu \mathrm{g} / \mathrm{dL}$

cuadrado, $t$ de Student, correlación y regresión lineal simple, usando el paquete estadístico SPSS versión 11,0 con un nivel de confianza de $95 \%$.

Para la realización del estudio se entregó un consentimiento informado representativo a los padres de familia y se contó con la autorización de la Sra. Norka Brios Ramos, directora del Centro Educativo No 5045 María Reiche.

\section{RESULTADOS}

La evaluación de la ansiedad fue realizada sólo a 69 niños; $60,9 \%$ de los niños mostró

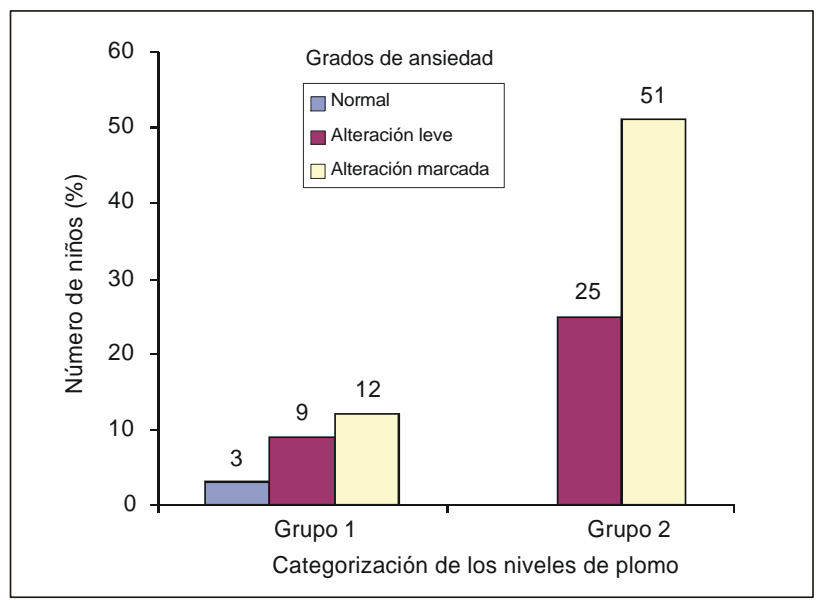

Grupo 1: menores o iguales a $30 \mathrm{mg} / \mathrm{dL}$ Grupo 2: mayores a $30 \mathrm{mg} / \mathrm{dL}$

Figura 3.- Grados de ansiedad según número de niños y categorización de los niveles de plomo. una ansiedad marcada (Figura 3), encontrándose significancia estadística entre los grados de ansiedad y la categorización de los niveles de plomo mediante la prueba de chi-cuadrado $(p=0,025)$ (Tabla 1). Además, se encontró diferencias significativas entre las medias del puntaje de la lista de chequeo de ansiedad y la categorización de los niveles de plomo mediante la prueba $t$ de Student ( $p=0,031)$ (Figura 4). De acuerdo al sexo, se encontró que $75 \%$ de las niñas y $56 \%$ de los niños presentaron una ansiedad marcada.

Se encontró una correlación inversa débil entre los niveles de plomo en sangre y los

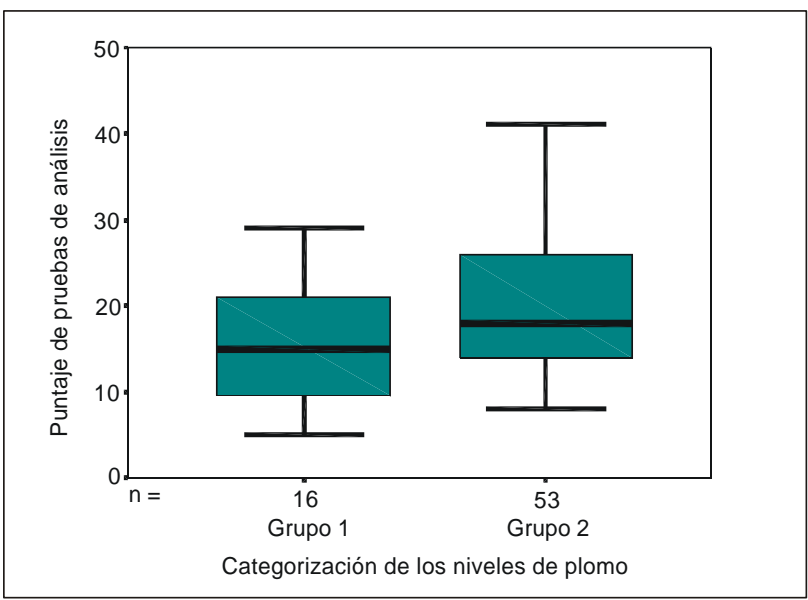

Grupo 1: menores o iguales a $30 \mathrm{mg} / \mathrm{dL}$ Grupo 2: mayores a $30 \mathrm{mg} / \mathrm{dL}$

Figura 4.- Puntajes de pruebas de ansiedad según categorización de los niveles de plomo. 
puntajes de las pruebas de habilidades motoras $(r=-0,26, p=0,03)$ (Figura 5).

Se encontró una correlación directa débil entre los puntajes de coeficiente intelectual y los puntajes de las pruebas de habilidades académicas $(r=0,314, p=0,009)$ (Figura 6).

No se encontró significancia estadística entre los puntajes de habilidades académicas y las concentraciones de plomo en sangre (pruebas $t$ de Student, correlación y regresión lineal simple); además, no se encontró significancia estadística entre la categorización de las habilidades académicas y la categorización de los niveles de plomo (prueba chi-cuadrado).

El $50 \%$ de los niños evaluados presentó una puntuación del coeficiente intelectual menor a lo normal; $77,1 \%$ de ellos tenía niveles de plomo superiores a $30 \mu \mathrm{g} / \mathrm{dL}$ en sangre; sin embargo, no se encontró significancia estadística entre las puntuaciones de las pruebas de coeficiencia intelectual y los niveles de plomo en sangre (pruebas $t$ de Student, correlación y regresión lineal simple); además, no se encontró significancia estadística entre la categorización de los puntajes de coeficiente intelectual y la categorización de los niveles de plomo en sangre (prueba chi-cuadrado).

La evaluación del IMC evidenció que 52,9\% no se encontraba dentro de los rangos normales; de éstos, $72,9 \%$ presentó niveles de plomo mayores a $30 \mu \mathrm{g} / \mathrm{dL}$ en sangre; sin embargo, no se encontró significancia estadística entre el IMC y la concentración de plomo en sangre (pruebas $t$ de Student, correlación y regresión lineal simple); así también, no se encontró significancia estadística entre la categorización del IMC y la categorización de los niveles de plomo (prueba chi-cuadrado).

Los resultados de las pruebas de seguimiento de instrucciones, habilidades comunicativas y manipulativas mostraron que $71,4 \%$ de los evaluados se ubicaba dentro de la categoría de desarrollo normal.

\section{DISCUSIÓN}

Investigaciones epidemiológicas han revelado que la intoxicación plúmbica crónica se asocia al deterioro de las funciones cognitivoconductuales y a alteraciones del desarrollo de los niños $\left({ }^{22}\right)$.

Se encontró relación entre los niveles de plomo y los grados de ansiedad, semejándose a los resultados obtenidos en el estudio de Needleman $\left.{ }^{(23}\right)$ en varones de una escuela pública en Pittsburg, entre las edades de 7 y 11 años, donde se observó que, con el pasar de los años, según aumentaba la cantidad acumulada de plomo aumentaban los reportes de agresividad, ansiedad y depresión.

Existen otros factores que pueden influir en el grado de la ansiedad, los cuales no pudieron ser controlados en la investigación; además, no se contó con individuos no intoxicados que pudieran ser comparados con el grupo de estudio. Por consiguiente, lo anteriormente mencionado podría afectar la relación encontrada entre los niveles de plomo y grado de ansiedad.

Taboada $\left({ }^{24}\right)$ sostiene que la prevalencia de trastornos de ansiedad en la infancia es más alta en las niñas que en los niños, concordando con los resultados obtenidos en el presente estudio (75\% de las niñas presentó ansiedad marcada).

Con respecto a las habilidades motoras, los resultados se asemejan a los obtenidos en Tijuana, donde se encontró una correlación inversa entre los niveles de plomo en sangre y las habilidades motoras $\left({ }^{17}\right)$.

La asociación entre la categorización de los niveles de plomo y los puntajes de la prueba de habilidades académicas no presentó significancia estadística; sin embargo, en un estudio hecho en el Callao se pudo observar una asociación inversa entre los niveles de plomo y rendimiento académico $\left({ }^{25}\right)$.

No se encontró una asociación significativa entre el coeficiente intelectual y la categorización de los niveles de plomo; esto difiere de los re- 


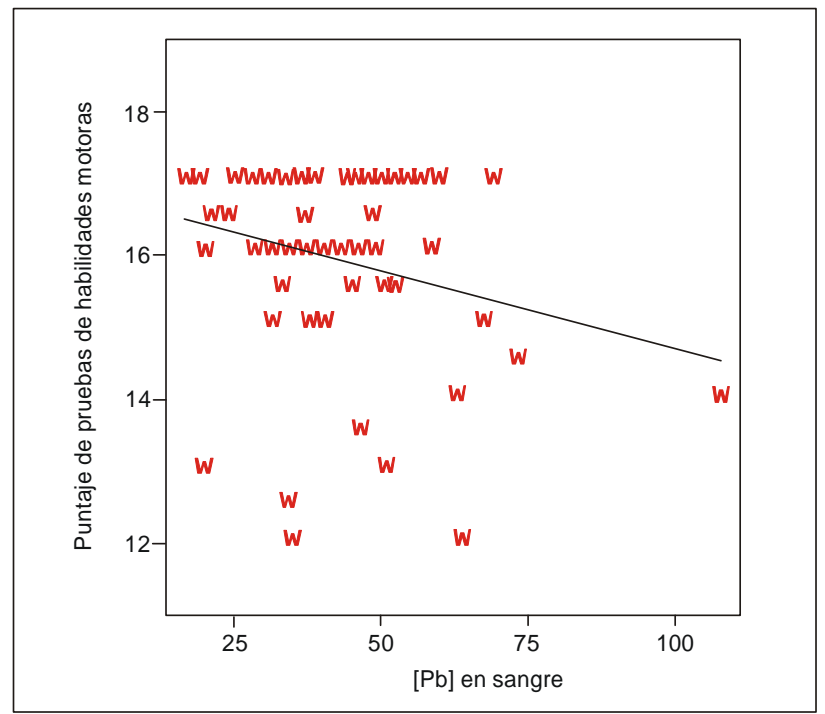

Ecuación de Regresión

Puntaje de pruebas de habilidades motoras

$=16,89+(-0,02)[\mathrm{Pb}]$ en sangre

$r=-0,264 ; r^{2}=0,07$

Figura 5.- Ecuación de regresión según puntajes obtenidos en pruebas de habilidades motoras y niveles de plomo en la sangre.

sultados obtenidos en Inglaterra $\left({ }^{26}\right)$, donde se encontró evidencia de un déficit en el coeficiente intelectual en los niños intoxicados; así también, Dietrich $\left({ }^{27}\right)$ reportó que hay una disminución de 8 puntos en el coeficiente intelectual al aumentar los niveles de plomo en sangre de 10 a $35 \mu \mathrm{g} / \mathrm{dL}$. Winneke $\left({ }^{28}\right)$ manifestó que niveles de plomo entre 10 y $20 \mu \mathrm{g} / \mathrm{dL}$ están asociados con la disminución de 1 a 3 puntos en el coeficiente intelectual. No se contó con individuos no intoxicados (controles) y esto podría explicar la diferencia de nuestros resultados con otros estudios.

Respecto al índice de masa corporal, no guardó asociación significativa con la categorización de los niveles de plomo; sin embargo, un estudio realizado en el Callao mostró una relación inversa entre el aumento de niveles de plomo en sangre y la disminución del IMC $\left({ }^{20}\right)$.

Se recomienda realizar controles anuales que evalúen el crecimiento y desarrollo cognitivoemocional. Además, la creación de programas

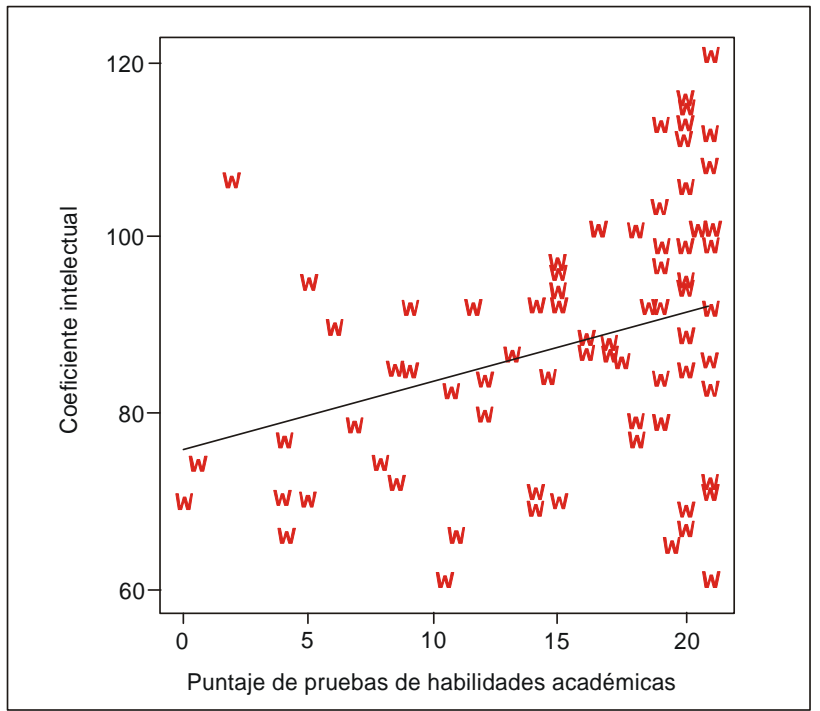

Ecuación de Regresión

Puntaje de pruebas de habilidades académicas

$=6,76+(0,11)$ Coeficiente intelectual

$r=0,316 ; r^{2}=0,09$

Figura 6.- Ecuación de regresión según puntajes obtenidos en pruebas de habilidades académicas y coeficiente intelectual.

de apoyo psicológico y estimulación académica para los niños del Centro Educativo N ${ }^{\circ} 5045$ "María Reiche".

Se debe realizar estudios para la determinación de lineamientos y normas que fijen los niveles de plomo aceptables en la población, ya que el Perú no cuenta con los mismos.

\section{BIBLIOGRAFÍA}

1. Ignacio A. Intoxicación por plomo en pediatría. Arch Pediatr Urug 2001; 72 (2): 133-8.

2. Corey G, Galvao L. Plomo. México DF: Metepec; 1989.

3. Goodman A, Ruddon W, Molinoff B, Limbird E, Hardman G. Las bases farmacológicas de la terapéutica 9ª ed. México DF: McGraw-Hill Interamericana; 1998.

4. Harrison. Principios de medicina interna. $15^{\mathrm{a}}$ ed. España: McGraw-Hill Interamericana; 2002.

5. Bertram G, Katzung M. Farmacología básica y clínica $8^{\text {a }}$ ed. México DF: El Manual Moderno; 2002.

6. Dirección General de la Salud Ambiental. Estudio de Plomo en sangre en una población seleccionada de la Oroya, Lima: Dirección General de la Salud Ambiental; 1999. 
7. Lugo M. Manejo clínico de las intoxicaciones por plomo. Segundo Simposium de actualización para atención a la población expuesta ambientalmente al plomo. México 2000.

8. Sanín L, González-Cossío T, Romieu I, Hernández-Avila M. Acumulación de plomo en hueso y sus efectos en la salud. Salud Pública Mex 1998; 40: 359-68.

9. Nordberg G, Mahaffey K, Fowler B. Introduction and summary. International workshop on lead in bone: Implications for dosimetry and toxicology. Environ Health Perspect 1991; 91: 3-7.

10. Gerhardsson L, Attewell R, Chettle D, Englyst V, Lundström N, Nordberg G, et al. In vivo measurements of lead in bone in long-term exposed lead smelters workers. Arch Environ Health 1993; 48: 147-56.

11. Carton J. Saturnismo. Med Clin (Barc) 1988; 91: 538-40.

12. Roa J, Uribe $\mathbf{C}$. Intoxicaciones comunes no medicamentosas. $3^{\mathrm{a}}$ ed. Bogotá: Editorial Ascofame; 1999.

13. Rivas F, Fernández N. Exposición urbano no ocupacional al plomo y niveles sanguíneos en embarazadas y recién nacidos. [Tesis doctoral]. Mérido: Departamento de Farmacología y Toxicología, Facultad de Medicina, Universidad de los Andes; 1999.

14. De la Cruz FZ, Mota L, Coste J. Niveles de Plomo en Sangre en Habitantes de Viviendas con Inversores, en la Zona Urbana de Vega, República Dominicana en el periodo agosto 1998-marzo 1999. [Tesis doctoral]. República Dominicana: Escuela de Medicina, Facultad de Ciencias de la Salud, Universidad Tecnológica del Cibao; 1999.

15. Instituto Nacional de Salud Pública. Intoxicación por plomo: de la detección a la prevención primaria. Salud Pública Mex 1995; 37 (3): 264-75.

16. Comité en Salud Ambiental de la Academia Norteamericana de Pediatría. Intoxicación con plomo: los niños afectados presentan síntomas neurológicos. Salud Pública Mex 2000; 30 (1): 233-40.

17. Centro Nacional de Salud Ambiental Enfermedades. Detectan altos niveles de plomo en la sangre de los niños. Salud Pública Mex 1999; 30 (2): 145-53.

18. Centers for Disease Control. Preventing Lead Poisoning in Young Children. Atlanta. US Departament of Health and Human Services; 1991.
19. Narciso J, Gaztañaga C, Espinoza R, Sánchez C, Moscoso S, Quequejana J, Hernández M. Estudio para determinar las Fuentes de Exposición de Plomo en la Provincia Constitucional del Callao, Perú. Activity Report $\mathrm{N}^{\circ}$ 104. Perú: Environmental Health Project; 2000 June. Contract No. HRN-4- 00- 99 00011- 00.

20. Hernández M, Wegner S. Estudio demográfico de salud de niños intoxicados por plomo tercer dosaje A.A.H.H "Puerto Nuevo"- Callao. Perú: Dirección General de Salud Ambiental. Feb 2002.

21. López J. Intoxicación por Plomo en Niños Menores de Seis Años en un Asentamiento Humano del Callao. An Fac Med Univ Nac Mayor San Marcos 2000; 61: 37-45.

22. Bellinger D, Levinton A, Waternaux $C$, Needleman $H$, Rabinowitz M. Longitudinal analyses of prenatal and postnatal lead exposure and early cognitive development. N Engl J Med 1987; 316: 1037-43.

23. Needleman HL, Riess JA, Tobin MJ, Biesecker GE, Greenhouse JB. Bone lead levels and delinquent behavior. JAMA 1996; 275 (5): 363-70.

24. Taboada J, Ezpeleta A. Trastornos por ansiedad en la infancia y adolescencia: Factores de riesgo. Ansiedad y Estrés 1998; 4 (1): 1-16.

25. Hernández M, Espinoza R, Moscoso S, Ortiz G, Lázaro B, Vigil L. Determinantes de los Niveles de Plomo en Sangre en Mujeres en el Posparto Temprano y Niños de 6 meses a Nueve años de Edad en Lima y Callao. Activity Report. N ${ }^{0}$ 72. Perú; Enviromental Health Project 1999. Contract No. HRN- C -03- 93- 00036- 11, Project No. 936- 5994.

26. Pocock SJ, Smith M, Baghurst P. Environmental lead and children's intelligence: a systematic review of the epidemiological evidence. BMJ 1994; 309: 1189-97.

27. Dietrich KN, Berger OG, Succop PA. Lead exposure and the motor developmental status of urban 6-years-old children in the Cincinnati prospective study. Pediatrics 1993; 91(2): 504-5.

28. Winneke G, Brockhaus A, Ewers U, Kramer U, Neuf M. Results from the European multicenter study on lead neurotoxicity in children: Implications for risk assessment. Neurotoxicity Teratol 1990; 12: 553-9. 